Advances in Dynamical Systems and Applications.

ISSN 0973-5321, Volume 16, Number 1, (2021) pp. 313-316

(C) Research India Publications

https://dx.doi.org/10.37622/ADSA/16.1.2021.313-316

\title{
Proof of Collatz Conjecture
}

\author{
Liu Chengning
}

Guangzhou Seventh Research Institute, China.

\begin{abstract}
This paper used the mathematical induction to prove Collatz Conjecture, namely, assumed that the conjecture of $3,5,7$, until $2 \mathrm{~m}+1$ were established, and the Collatz Conjecture transformation rule was used to prove that the conjecture was true whether $m$ in $2 m+3$ was odd or even, which thoroughly proved Collatz Conjecture.
\end{abstract}

Keywords: Integer Parity, Scaling, Mathematical Induction

\section{INTRODUCTION}

This paper used the mathematical induction to prove Collatz Conjecture, which is a mathematical issue that has not been completely resolved by public channels. The conjecture has been verified by computer to $7^{*} 10^{\wedge} 11$, therefore, people have sufficient reasons to believe that it is true. However, there is no convincing proof. After decades of hard work, the author first partially proved it in 2010 and got affirmed by mathematics professionals. After ten years of efforts, based on their suggestions, the author finally fully proved it, making Collatz Conjecture Collatz Theorem.

\section{MATERIALS AND METHODS}

This paper uses mathematical induction to prove the Collatz Conjecture. First, it verifies the conjecture of 3,5 and 7, and on this basis, it assumes that the conjecture of 3,5 and 7 is true until the conjecture of $2 m+1$ is true. Using the transformation rule of Collatz Conjecture, it proves that the conjecture of $2 m+3$ is true whether $m$ is odd or even, and completely proves the Collatz Conjecture . 
Collatz Conjecture refers that when $\mathrm{N}$ is even, perform $\mathrm{N} / 2$, when $\mathrm{N}$ is odd, perform $3 \mathrm{~N}+1$, the result will definitely be 1 in the end after such transformations.

Verification:

when $\mathrm{N}=3$,then, 10-5-16-8-4-2-1,

when $\mathrm{N}=5$, then, 16-8-4-2-1,

when $\mathrm{N}=7$, then, 22-11-34-17-52-26-13-40-20-10-5-16-8-4-2-1,

Obviously, it only needs to prove that Collatz Conjecture is true when $\mathrm{N}$ is odd. Set $\mathrm{N}$ to be greater than 7 , and $\mathrm{N}=2 \mathrm{~m}+1$. There are only two cases for $\mathrm{m}$, odd and even. First, prove Collatz Conjecture when $\mathrm{m}$ is odd. Because the conjecture is verified to be true when $\mathrm{m}$ is 3,5 , and 7 . So it can be assumed that when $\mathrm{m}$ is odd, i.e. $3,5,7 \ldots 2 \mathrm{~m}+1$, the conjecture is true, set $\mathrm{N}+2=2 \mathrm{~m}+3$. If Collatz Conjecture is still true for $2 \mathrm{~m}+3$, then when $\mathrm{m}$ is odd, Collatz Conjecture is true. (mathematical induction)

Because $2 m+3$ is odd, set $3 *(2 m+3)+1=6 m+10$ to be even, it is equal to $3 m+5$ after being divided by 2 , which is still even, set $3 m+5=2 k_{n}$,

( $\mathrm{k}$ is an exponent, $\mathrm{n}$ is odd)

When $\mathrm{m}$ is greater than $3,2 \mathrm{~m}+1>(3 \mathrm{~m}+5) / 2>(3 \mathrm{~m}+5) / 2^{2}>\ldots \ldots>(3 \mathrm{~m}+5) / 2^{\mathrm{k}}$, that is, $\mathrm{N}+2$ belongs to $3,5,7 \ldots \ldots .2 \mathrm{~m}-1$, the previous assumption stands, so Collatz Conjecture is true when $\mathrm{m}$ is odd.

If $\mathrm{m}$ is even, set $\mathrm{m}=2^{k} \mathrm{n}$, ( $\mathrm{n}$ is odd). At this time, assume that the conjecture stands for $3,5,7 \ldots 2 m+1$, set $N+2=2 m+3$,

when $\mathrm{N}+2=2^{k+1} \mathrm{n}+3$ is odd, set $3(\mathrm{~N}+2)+1=32^{k+1} \mathrm{n}+10$ to be even, after being divided by 2 , there is $32^{k} \mathrm{n}+5$, which is odd,

set $3\left(32^{k} n+5\right)+1=3^{2} 2^{k} n+16$ to be even, after being divided by 16 , there is $3^{2} 2^{k-4}$ $\mathrm{n}+1$, which is odd. Set $3\left(3^{2} 2^{k-4} \mathrm{n}+1\right)+1$,

$3^{3} 2^{k-4} \mathrm{n}+4$ is even, after being divided by 4 , there is $3^{3} 2^{k-6} \mathrm{n}+1$, which is odd. Set $3\left(3^{3} 2^{k-6} n+1\right)+1=3^{4} 2^{k-6} n+4$ to be even, after being divided by 4 , there is $3^{4} 2^{k-8}$ 
$\mathrm{n}+1$, which is odd. Set $3\left(3^{4} 2^{k-8} \mathrm{n}+1\right)+1$, namely, $3^{5} 2^{k-8} \mathrm{n}+4$ to be even, after being divided by 4 , there is $3^{5} 2^{k-10} n+1$,

After the calculation, $3^{6} 2^{k-12} \mathrm{n}+1,3^{7} 2^{k-14} \mathrm{n}+1 \ldots \ldots$

It is found that the calculation is regular, that is, every time the exponent of 3 increases by 1 , the exponent of 2 decreases by 2 . If the exponent of 3 is $r$, then $3^{r} 2^{k-}$ $2 r n+1$, because every time when it is multiplied by 3 , the exponent of 3 increases by 1 , and when it is divided by $2^{2}$, the exponent of 2 decreases by 2 . The process can be repeated for a limited number of times.

$\mathrm{k}-2 \mathrm{r}$ can only be 0,1 , when $\mathrm{k}$ is even, it can be 0 , when $\mathrm{k}$ is odd, it can be 1 . Set $\mathrm{k}=2 \mathrm{r}$, then $3^{r} \mathrm{n}+1$ is even.

Set $3^{r} n+1=2^{i} t$,

$2 \mathrm{~m}+1=2^{2 r+1} \mathrm{n}+1>\left(3^{r} \mathrm{n}+1\right) / 2>\left(3^{\mathrm{r}} \mathrm{n}+1\right) / 2^{2}>\left(3^{\mathrm{r}} \mathrm{n}+1\right) / 2^{i}$

That is, $\mathrm{N}+2$ belongs to $3,5,7 \ldots 2 \mathrm{~m}-1$, the previous assumption stands. So when $\mathrm{m}=$ $2^{k} \mathrm{n}, \mathrm{k}=2 \mathrm{r}$, Collatz Conjecture is true. If $\mathrm{k}=2 \mathrm{r}+1,3^{\mathrm{r}} 2 \mathrm{n}+1$ is odd, perform $3\left(3^{\mathrm{r}}\right.$ $2 n+1)+1=3^{r+1} 2 n+4$, after being divided by $2,3^{r+1} n+2$ is odd,

$3\left(3^{r+1} \mathrm{n}+2\right)+1$, that is, $3^{r+2} \mathrm{n}+7$ is even, set $3^{r+2} \mathrm{n}+7=2^{s} \mathrm{w}$

When $\mathrm{k}=2 \mathrm{r}+1, \mathrm{~m}=2^{\mathrm{k}} \mathrm{n}, 2 \mathrm{~m}+1=2^{2 \mathrm{r}+2} \mathrm{n}+1>\left(3^{r+2} \mathrm{n}+7\right) / 2>\left(3^{r+2} \mathrm{n}+7\right) / 2^{2}$ $>\left(3^{r+2} \mathrm{n}+7\right) / 2^{\mathrm{s}}$

Then $\mathrm{N}+2$ belongs to $3,5,7 \ldots 2 \mathrm{~m}-1$. The previous assumption stands, so when $\mathrm{m}=2^{k}$ $\mathrm{n}, \mathrm{k}=2 \mathrm{r}+1$, Collatz Conjecture is true. In summary, whether $\mathrm{m}$ is odd or even, Collatz Conjecture is true, this conjecture is proved. 


\section{RESULT AND DISCUSSION}

A result can be obtained from the proof above, that is, for any natural number $\mathrm{N}$, when it is even, perform $\mathrm{N} / 2$, when it is odd, perform $3 \mathrm{~N}+1,1$ will be definitely obtained in the end after such transformations. This paper only solved an isolated problem that had puzzled people for many years. Its application value still waits to be discovered in the future.

\section{CONCLUSION}

We can be pretty sure that Collatz Conjecture is true, which can be used as a theorem in the future.

\section{ACKNOWLEDGMENTS}

I would like to thank my wife Mo Guofeng, who took care of me when I was sick and enabled me to make significant discoveries after recovery.

I also would like to extend my thanks to Yang Zhihua, editor of Scientia Sinica (Mathematica), for his encouragement and support.

Thanks to everyone who helped me.

\section{REFERENCES}

[1] Loo-keng Hua, An Introduction to Number Theory, (Science Press, 1979)

[2] Min Sihe, Yan Shijian, Elementary Theory of Numbers, (Higher Education Press, 2003)

[3] Jin Ping, Li Xiuping, Hao Shuiping, 100 Basic Mathematical Questions, (Shanxi Science and Technology Press, 2004)

[4] Deng Shoucai, A Beauty Pageant and Appreciating of Interesting Elementary Number Theory, (Harbin Institute of Technology Press, 2015: 45-48)

[5] One Hundred Thousand Whys, (Juvenile and Children's Publishing House, 2003: 152-154) 